\section{Collision tumour of sigmoid colon - a rare presentation}

\section{Introduction}

Two histologically distinct tumours found coincidentally in the same location are known as collision tumours. They are rarely seen in the colon. The occurrence of collision tumours at different sites has been previously reported. The collision of adenocarcinoma and lymphoma of the sigmoid colon is rare. We present here such a case. The determination of the dominant tumour by immunohistochemistry is of utmost important for optimal management.

\section{Case report}

A 77-year-old male presented with painless bleeding per rectum of 10 days duration. The stool was mixed with blood and mucus with a frequency of twice a day. This was accompanied by mild abdominal pain but no sensation of an abdominal lump. There

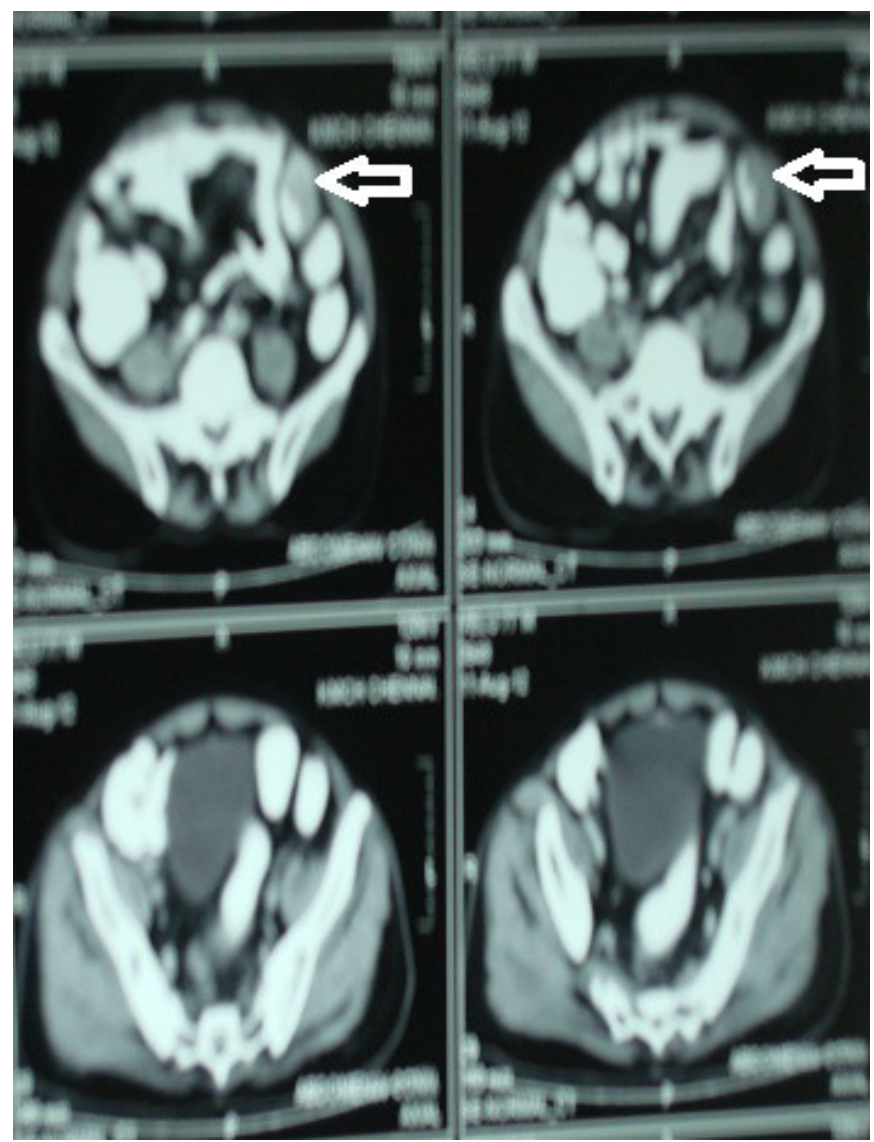

Figure 1: Computed tomography scan of the abdominal mass (arrows) was no tenesmus or urgency during defecation. The patient had quit smoking and alcohol a few years ago. There was no significant loss of appetite or loss of weight, and there was no family history of gastrointestinal cancers. Examination revealed a BMI of 18.5. Abdomen and per rectal examination revealed no abnormality. Blood reports were normal except for low haemoglobin at $10 \mathrm{~g} / \mathrm{dl}$. Contrast enhanced computed tomography of the abdomen revealed an endoluminal mass in the sigmoid colon with abdominal lymphadenopathy (Figure 1). Sigmoidoscopy revealed a $3 \times 3 \mathrm{~cm}$ circumferential ulceroproliferative friable growth in the sigmoid colon with multiple erythematous patches in the rectum. Multiple biopsies were taken (Figure 2). Histopathology revealed features suggestive of mixed, poorly differentiated adenocarcinoma and lymphoma (Figure 3). So immunohistochemistry was performed which showed diffuse nuclear reactivity with anti-CD45 antibody (Figure 4) and focal nuclear reactivity with anti-CD3 antibody (Figure 5), confirming the diagnosis of a high-grade, B cell, non-Hodgkin's lymphoma. The patient was referred for chemotherapy and after receiving two cycles of chemotherapy the patient is doing well.

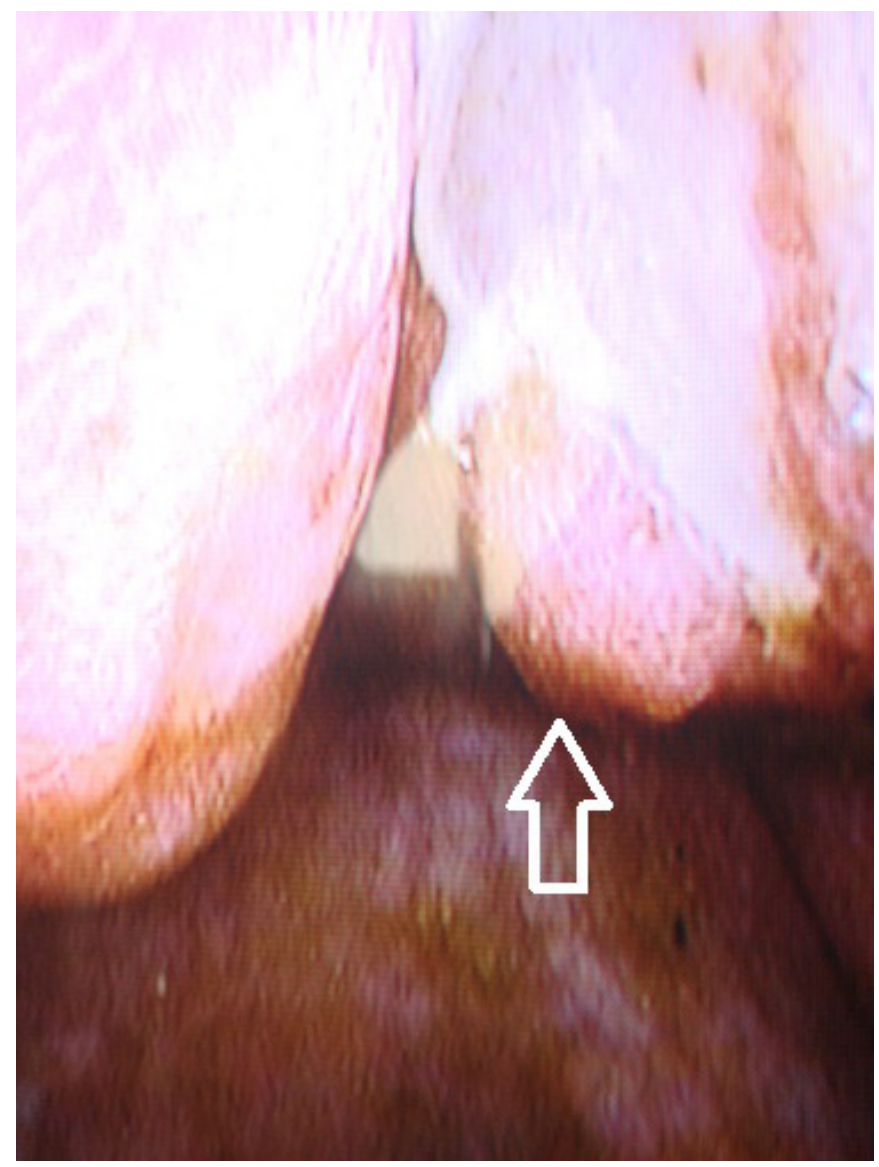

Figure 2: Sigmoidoscopic appearance of the neoplastic mass 


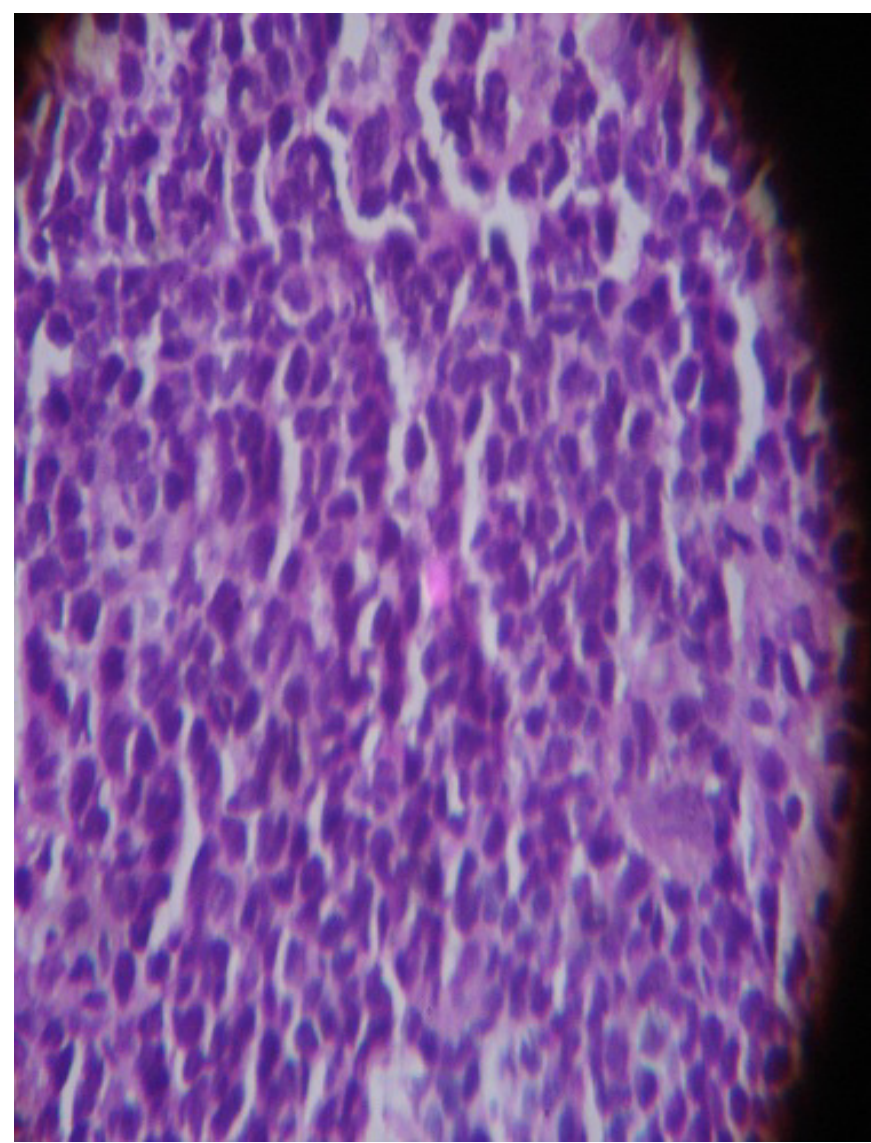

Figure 3: Histopathology of the neoplastic mass showing an admixture of poorly differentiated adenocarcinoma and lymphoma (hematoxylin and eosin, 40x)

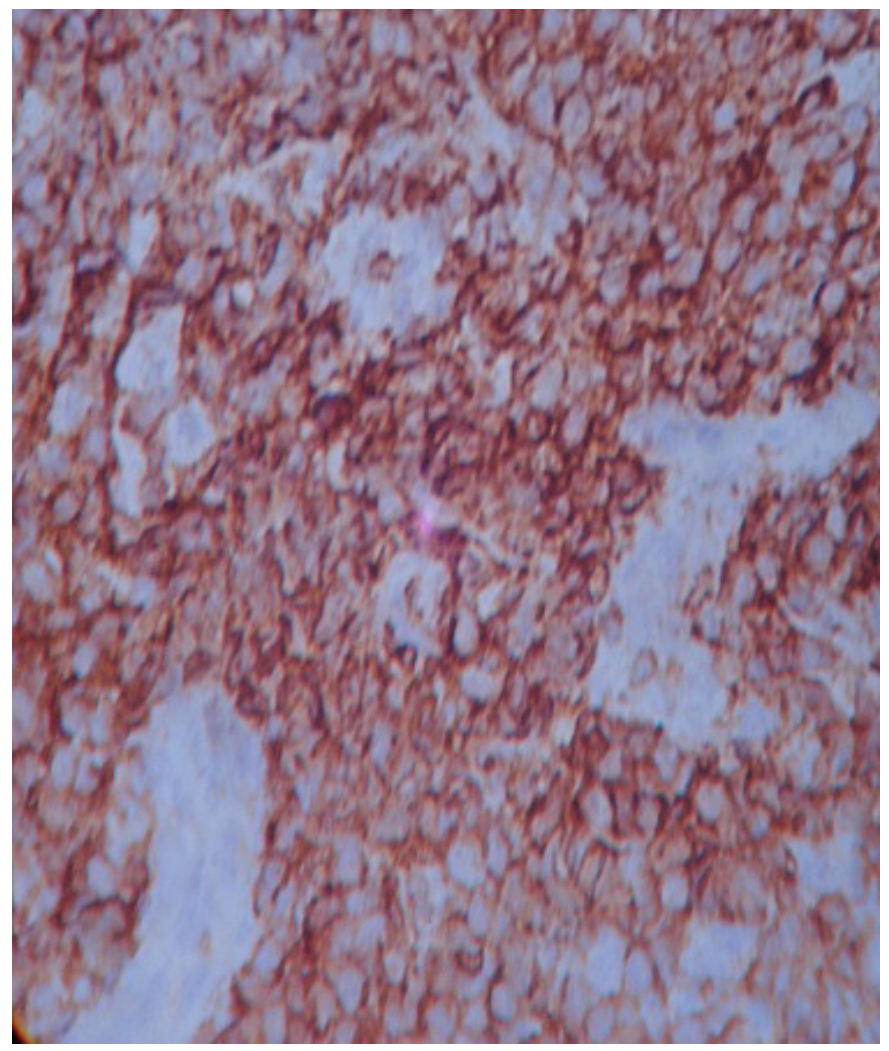

Figure 4: Immunohistochemistry of the neoplasm showing features of non-Hodgkin's lymphoma characterized by diffuse nuclear reactivity stained with anti-CD45 antibody (40x)

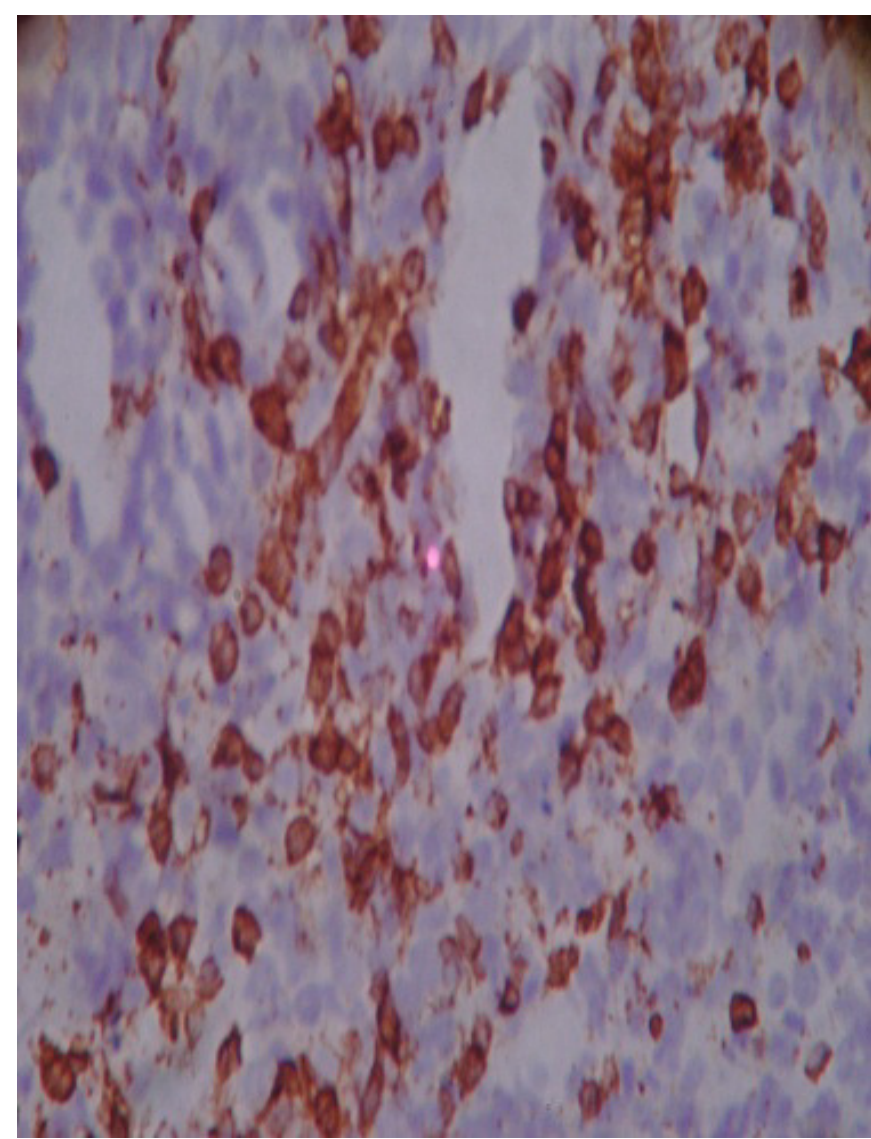

Figure 5: Immunohistochemistry of the neoplasm showing features of non-Hodgkin's lymphoma marked by focal nuclear reactivity stained with anti-CD3 antibody (40x)

\section{Discussions}

The most common malignant neoplasm of the colon is adenocarcinoma colon. Collision tumours comprising of two independent tumour phenotypes can also rarely occur in the colon. They can be a combination of adenocarcinoma with carcinoid tumour, ${ }^{1}$ transitional cell carcinoma, ${ }^{2}$ or lymphomas. ${ }^{3-}$ ${ }^{6}$ Although the formation of collision tumours is not well understood, theories explaining their occurrence include: 1) simultaneous proliferation of two different cell lines, 2) common origin from pluripotent cancerous stem cell which differentiates into two components, and 3) chance apposition of two unrelated tumours. It is difficult to predict the biological behaviour of collision tumours. The final outcome is determined by the dominant tumour, although whether the more histologically aggressive component or the more predominant/voluminous component determines the outcome is still unclear. Immunohistochemistry and genetic analysis help identify the dominant component. ${ }^{\text {? }}$

Only a few cases of colo-rectal collision tumours comprising of lymphoma and adenocarcinoma been reported previously. Sasaki et al reported a cecal collision tumour consisting of 
primary malignant lymphoma and adenocarcinoma. ${ }^{8}$ Chang et al reported a collision tumour made up of a diffuse large B cell lymphoma at the base of skull and in the ileocecal region. ${ }^{9}$ Minato et al reported a primary malignant lymphoma and poorly differentiated adenocarcinoma collision tumour of the ascending colon. ${ }^{10} \mathrm{Lu}$ et al reported a collision tumour of rectal lymphoma and adenocarcinoma and highlighted the need for clinical awareness of such tumours and to plan treatment strategies depending on the biological aggressiveness of each tumour component. ${ }^{11}$

Histopathology in our patient suggested the presence of adenocarcinoma and lymphoma in our patient, but immunohistochemistry helped identify the dominant tumour and guide further management.

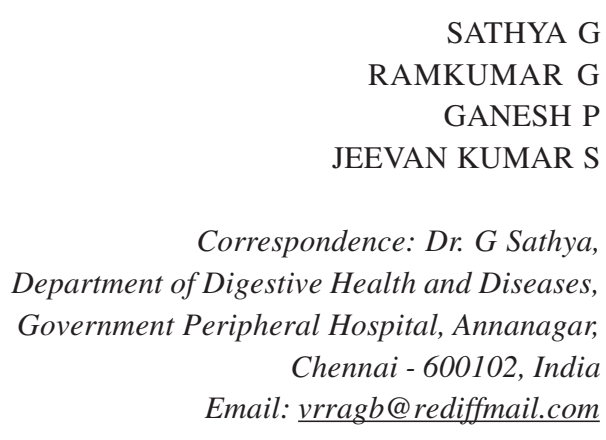

\section{References}

1. Olinici CD, Domsa I, Crisan D. Collision adenocarcinomacarcinoid tumor of the colon. Case report and review of the literature. Rom J Morphol Embryol. 2007;48:75-8.

2. Krausz M, Manny J, Aviad I, Charuzi I. Vesicocolic fistula due to "collision" between adenocarcinoma of the colon and transitional cell carcinoma of the urinary bladder. Cancer. 1976;38:335-40.

3. Takafumi A, Mitsuru S, Daisuke I, Fuyuki I, Hiroyuki S. Coexistence of primary colonic malignant lymphoma and rectal adenocarcinoma. A case report and common clinical features in literatures. Jpn J Gastroenterol Surg. 2001;34:54-8.

4. Chazouilleres O, Andreani T, Boucher JP, Calmus Y, de Sigalony $\mathrm{H}$, Nordlinger R, et al. [Rectal adenocarcinoma in association with lymphoma (“collision tumor”)]. Gastroenterol Clin Biol. 1990;14:185-6.

5. Mir-Madjlessi SH, Vafai M, Khademi J, Kamalian N. Coexisting primary malignant lymphoma and adenocarcinoma of the large intestine in an IgA-deficient boy. Dis Colon Rectum. 1984;27:822-4.

6. Wagle SD, Mohandas KM, Vazifdar KF, Dhir V, Swaroop VS, Jagannath P, et al. Synchronous adenocarcinoma and lymphoma of the colon. Indian J Gastroenterol. 1997;16:28-9.

7. Brahmania M, Kanthan CS, Kanthan R. Collision tumor of the colon—colonic adenocarcinoma and ovarian granulosa cell tumor. World J Surg Oncol. 2007;5:118.
8. Sasaki S, Hatanaka K, Sahara N, Uekusa T, Hirayama K, Shirahata A, et al. Collision tumor of primary malignant lymphoma and adenocarcinoma in the colon: report of a case. Surg Today. 2010;40:975-81.

9. Chang H, Chuang WY, Shih LY, Tang TC. Collision in the colon: concurrent adenocarcinoma and diffuse large B-cell lymphoma in the same tumour. Acta Clin Belg. 2011;66:302-4.

10. Minato E, Fujino I, Sugihira N, Matsumoto K, Shima K. A case of collision tumor of primary malignant lymphoma and poorlydifferentiated adenocarcinoma in the ascending colon. Jpn J Gastroenterol Surg. 2004;37:213-6.

11. Lu P, Kong XL,Yu JY, Hong L, Ning HY, MengYH. Rectal lymphoma and adenocarcinoma (collision tumor): a clinicopathological analysis. Chin J Diag Pathol. 2012:111-14.

\section{Hepatitis A induced hemophagocytic syndrome}

\section{Introduction}

Hemophagocytic lympho histiocytosis (HLH) is a disorder of uncontrolled, pathologic activation of the immune system leading to extreme hyperinflammation. HLH could be familial (due to genetic defects leading to impaired function of natural killer and cytotoxic Tcells) or acquired. Acquired forms of HLH are usually encountered as aresult of viral infections. ${ }^{1}$ Acquired HLH due to viral infections (also known as virus associated hemophagocytic syndromeor VAHS) is most commonly associated with Ebstein Barr virus (EBV), cytomegalovirus (CMV), human herpesvirus type 6 (HHV-6) and HHV-8. Other rarely implicated viruses are human immunodeficiency virus (HIV), adenovirus, coxsackie virus, measles, dengue, rubella, parvovirus B19, herpes simplex virus and varicella zoster virus. ${ }^{2}$ The presence of persistent high fever, peripheral blood cytopenia (two or more series), hyperferritinemia, hyperlipidemia, hemophagocytosis in the bone marrow (hemophagocytic cell ratio over 3\%), splenomegaly and/or lymph nodeenlargement along with active viral infection are required to confirm the diagnosis of VAHS. ${ }^{3}$ Despite the high incidence of hepatitis A virus (HAV) infection in pediatric population, HAV induced VAHS has rarely been described in literature. ${ }^{2}$ We report a case of 15 -year-old male child with 